\title{
Radiosurgery for Intracranial Aneurysms: Blitzkrieg or Guerrilla War?
}

\author{
Manjul Tripathi \\ Department of Neurosurgery, Neurosurgery Office, Post Graduate Institute of Medical Education and \\ Research, Chandigarh, India
}

I read with great interest the article "Gamma knife radiosurgery of digital aneurysm: A case series" by Liscak et al. [1]. The article details the successful obliteration of bleeding distal aneurysms not associated with arteriovenous malformation (AVM) with gamma knife radiosurgery (GKRS). The aneurysms were treated by targeting a single isocenter centralizing over the aneurysm with a peripheral dose of 24-29 Gy. The authors concluded that GKRS might serve as a safe, minimally invasive technique in the treatment of selected distal aneurysm [1].

Since the introduction of radiosurgery for AVM, neurosurgical and radiosurgical communities remained curiously enthusiastic to expand the horizon of radiosurgery by targeting other vascular anomalies such as cavernous malformations, fistulas, and now aneurysms. Unfortunately, the spectacular success with AVM could not be reproduced with other intracranial vascular pathologies $[2,3]$. Among all vascular anomalies, aneurysm is considered to be the most ferocious and morbid condition with the potential of significant chances of rebleeding and complications including high rates of death. Aneurysms in traditionally remote areas of the brain, such as mycotic aneurysms, and complex intranidal and perinidal aneurysms with AVM practically fall in no

karger@karger.com

(c) 2021 S. Karger AG, Basel

www.karger.com/sfn

Karger! man's land. The natural history of these complex aneurysms has been significantly different from the natural history of an isolated ruptured berry aneurysm. Both surgery and endovascular interventions frequently remain limited to sacrifice of the parent artery with variable outcomes.

A few animal experimental studies have hypothesized about the efficacy of GKRS in the management of aneurysm treatment. GKRS showed a linear longitudinal decrease in total and luminal volume with promising changes similar to those seen in the AVMs [4]. Apart from the difference in the vascular wall, the differential response of AVMs and aneurysms to radiosurgery is because of the differences in their neighborhood. Whereas an AVM enjoys connective tissue stroma rich in spindleshaped cells and myofibroblasts, an aneurysm bathes in the cerebrospinal fluid. This rapid activation of myofibroblasts in the connective tissue stroma helps in hyalinization of the occluded vessel periphery, further stabilizing the partially obstructed AVM vessels under the influence of radiosurgery. Contrary to this, aneurysms lack any such supporting surroundings and have to depend on gradual intraluminal thrombosis for radiosurgery to be of any useful impact [5]. 
In the present series, it could not be ascertained if the disappearance of aneurysm was because of radiosurgery or secondary to obliteration of the feeder artery [1]. In view of the differential natural history and mortality profile of distal aneurysms from saccular aneurysms on the circle of Willis, it remains imperative to analyze the obliteration rate of an aneurysm after radiosurgery. With the current evidence, it cannot be concluded if radiosurgery actually helps an aneurysmal obliteration with direct impact. Until autopsy studies provide pathological evidence and natural history takes a favorable turn under the influence of radiosurgery, the question of its efficacy would loom large. Hopefully, in the coming times, it would be exciting to know the role of radiosurgery as a primary or adjunctive treatment option in the management of this difficult entity.

\section{Conflict of Interest Statement}

The author has no conflicts of interest to disclose.

\section{Funding Sources}

The authors have no conflicts of interest to disclose.

\section{Author Contributions}

M.T.: concept, original draft and review, and administrative support.

\section{References}

1 Liscak R, Vymazal J, Chytka T. Gamma knife radiosurgery of distal aneurysm: a case series. Stereotact Funct Neurosurg. 2021:1-6.

2 Mukherjee KK, Kumar N, Tripathi M, Oinam AS, Ahuja CK, Dhandapani S, et al. Dose fractionated gamma knife radiosurgery for large arteriovenous malformations on daily or alternate day schedule outside the linear quadratic model: proof of concept and early results. A substitute to volume fractionation. Neurol India. 2017;65(4):826-35.
3 Tripathi M. Radiosurgery for dural arteriovenous fistulas: bet on the jockey, not on the horse. Neurol India. 2020;68(4):821-3.

4 Meadowcroft MD, Cooper TK, Rupprecht S, Wright TC, Neely EE, Ferenci M, et al. Gamma Knife radiosurgery of saccular aneurysms in a rabbit model. J Neurosurg. 2018;129(6): 1530-40.

5 Tripathi M, Batish A, Mohindra S. Gamma Knife radiosurgery for berry aneurysms: quo vadis. J Neurosci Rural Pract. 2021;12(1): $182-4$. 